\title{
Hong Kong: recent development of mental health services
}

\author{
Wai-chi Chan, Linda Chiu-wa Lam \& Eric Yu-hai Chen
}

\begin{abstract}
SUMMARY
From a very deprived baseline, mental health services in Hong Kong have undergone some growth over the past decade. The number of inpatient beds for psychiatric treatment dropped by one-third, but the community mental health service expanded significantly. A number of low-resource phase-specific and age-specific treatment programmes were introduced, with promising initial results. However, there remain many challenges, including a serious shortage of well-trained mental health professionals. Much more investment in mental health workforce development is required to meet the service needs not only of today, but also of tomorrow.
\end{abstract}

\section{DECLARATION OF INTEREST}

None

Following British rule from 1842 to 1997 , Hong Kong is now a Special Administrative Region of China. The mental health system has largely remained unchanged since it acquired this status. The Hospital Authority, a statutory governmentfunded body, is the major provider of mental healthcare for a population of over 7 million. The Mental Health Ordinance of Hong Kong, which was modelled on the Mental Health Act of the UK, still guides the mental health provision locally.

\section{From hospitals to community}

Perhaps the most significant change in mental healthcare in Hong Kong over the past decade is the dramatic transition from hospital-based to community-based services (Hospital Authority 2001, 2013a). The number of psychiatric beds under the Hospital Authority peaked in 2000, when there were 5395 beds distributed across two psychiatric hospitals and ten general hospitals. The size of in-patient facilities then started to decrease. According to the latest Hospital Authority statistics, there were 3607 psychiatric beds in 2012, representing a reduction of 33\% over the past 12 years (Hospital Authority 2013a). Meanwhile, psychiatric out-patient attendances have been increasing steadily from 432046 in 2000 to 755745 in 2012 - a rise of almost $75 \%$. Psychiatric day hospital attendances also increased, from 146729 in 2000 to 220532 in 2012 , a rise of around $50 \%$. In addition, community psychiatric services provided 42756 home visits in 2000. Twelve years later, 198354 home visits were made to patients receiving community psychiatric services.

In line with the enhancement of community psychiatric services, a number of programmes specific to disease phases have been introduced over the past few years. A notable example is the Early Assessment Service for Young People with Psychosis (EASY) programme, which consists of public awareness, early detection and specialised case management. A recent cohort study showed that patients in the EASY group had longer fulltime employment or study, fewer days of hospital admissions, fewer suicides and disengagements from services than the historical control group (Chen 2011). Specialised treatment programmes catering for different age-specific disorders, for instance, the Elderly Suicide Prevention Programme, have also been developed.

\section{Challenges}

Although there have been exciting developments, we are facing significant challenges in various areas. Some of these challenges are not new to mental health professionals from other parts of the world, for example the prevailing stigma towards mental health problems, fragmentation of healthcare system and a lack of dedicated mental health funds. But others are less frequently encountered by our overseas counterparts. For example, crowded living conditions in Hong Kong have posed unexpected difficulty in the delivery of psychiatric treatment in the community. By mid2012, the population density of Hong Kong stood at a staggering 6620 persons per square kilometre (Information Services Department 2013). It is drastically higher than in most high-income countries/regions such as Japan $\left(351 / \mathrm{km}^{2}\right)$, the UK $\left(259 / \mathrm{km}^{2}\right)$ and the USA $\left(34 / \mathrm{km}^{2}\right)$ (World Bank 2014). The overcrowded living conditions have made family members unwilling or unable
Wai-chi Chan is a Clinical Associate Professor in the Department of Psychiatry at the University of Hong Kong. Linda Chiu-wa Lam is a Clinical Professor and Chairman of the Department of Psychiatry at the Chinese University of Hong Kong. Eric Yu-hai Chen is a Professor and Head of the Department of Psychiatry at the University of Hong Kong. Correspondence Professor Eric Yu-hai Chen, Professor and Department Head, Department of Psychiatry, University of Hong Kong, 2/F, New Clinical Building, Queen Mary Hospital, Pokfulam, Hong Kong SAR. Email: eyhchen@hku.hk 
to support patients in the community, especially during periods when they need more intensive care. Besides, at least some patients find hospital care a desirable alternative when compared with their own accommodation.

Another significant challenge in Hong Kong is the inadequate involvement of primary care services in the provision of mental healthcare. Unlike health systems in countries such as the UK, a large proportion of primary care in Hong Kong is provided by general practitioners in the private sector. It is therefore not surprising that the public specialist psychiatric services operated by the Hospital Authority, which is heavily subsidised by the government, draw most of the patients. This is reflected in the steady rise in the number of patients receiving the Hospital Authority psychiatric services (including inpatient, specialist out-patient, day hospital and psychiatric community services) from 147557 in 2007-2008 to 186907 in 2011-2012, an increase of $26 \%$ (Hong Kong Government Information Centre 2012). The unsatisfactory interface between primary care and specialist psychiatric services has made the latter overloaded. The waiting time for psychiatric services has therefore been lengthening over the years: in routine cases in 2013 , around $90 \%$ of individuals waited 81 weeks for a first appointment at a psychiatric out-patient clinic (Hospital Authority 2013b). Patients, while paying a relatively low out-of-pocket consultation fee, are giving their 'precious time' in order to obtain proper psychiatric care.

One would not expect a shortage of mental healthcare staff in a prosperous city like Hong Kong. However, one of the most significant challenges to local psychiatric services is actually an insufficient number of well-trained mental health professionals. According to the latest Mental Health Atlas (World Health Organization 2011), there were only 4.39 psychiatrists per 100000 population in Hong Kong. This was well below other high-income regions such as England (17.65/100 000) and Japan (10.1/100 000). The size of the nursing workforce was also relatively small in Hong Kong: 29.15 mental health nurses per 100000 population. Contrast this with 83.23 per 100000 and 102.55 per 100000 in England and Japan respectively. With only 0.58 psychologists (England: 12.84; Japan: 3.99) and 2.26 occupational therapists (England: 28.72; Japan: 4.85) per 100000 population in Hong Kong, we are facing a shortage of other mental health professionals too. The recent introduction of new psychiatric programmes, which demand additional case managers, has intensified the staffing strain across the territory. Workforce shortage is anticipated to increase unless longterm commitment is undertaken to improve the training, recruitment and retention of mental health professionals.

\section{References}

Chen EYH, Tang JYM, Hui CLM, et al (2011) Three-year outcome of phase-specific early intervention for first-episode psychosis: a cohort study in Hong Kong. Early Intervention in Psychiatry, 5: 315-323.

Hong Kong Government Information Centre (2012) Legislative Council 04: Number of Persons Receiving Psychiatric Services Provided by the Hospital Authority (HA). Hong Kong Government (http://gia.info.gov.hk/ general/201205/30/P201205300297_0297_94676.pdf).

Hospital Authority (2001) Hospital Authority Statistical Report 1999-00. Hospital Authority (http://www.ha.org.hk/upload/publication_15/114. pdf).

Hospital Authority (2013a) Hospital Authority Statistical Report 2011-2012. Hospital Authority (http://www.ha.org.hk/upload/ publication_15/471.pdf).

Hospital Authority (2013b) Chief Executive's Progress Report on Key Performance Indicators (KPIS) (AOM-P1003). Hospital Authority (https:// www.ha.org.hk/haho/ho/cad_bnc/AOM-P1003.pdf).

Information Services Department (2013) Hong Kong: The Facts. Population. Information Services Department, Hong Kong SAR Government (http://www.gov.hk/en/about/abouthk/factsheets/docs/ population.pdf)

World Bank (2014) Population density (people per sq. km of land area). World Bank Group (http://data.worldbank.org/indicator/EN.POP.DNST)

World Health Organization (2011) Mental Health Atlas 2011. WHO. 